\title{
ON THE PRODUCTS OF FUNCTIONS REPRESENTED AS CONVOLUTION TRANSFORMS
}

\author{
I. I. HIRSCHMAN, JR., AND D. V. WIDDER
}

The authors have in a number of papers, $[1],[2]$, and $[3],{ }^{1}$ considered the convolution transforms

$$
f(x)=\int_{-\infty}^{\infty} G(x-t) d \alpha(t)
$$

with kernels of the form

$$
\begin{aligned}
& G(t)=\frac{1}{2 \pi i} \int_{-i \infty}^{i \infty} E(s)^{-1} e^{s t} d s, \\
& E(s)=e^{b s} \prod_{k=1}^{\infty}\left(1-\frac{s}{a_{k}}\right) e^{s / a_{k}} .
\end{aligned}
$$

Here $b,\left\{a_{k}\right\}_{1}^{\infty}$ are real constants subject only to the restriction that $\sum_{1}^{\infty} a_{\mathbf{k}}^{-2}<\infty$. Let $\lambda_{1}$ and $\lambda_{2}$ be strictly positive real numbers such that $\lambda_{1}+\lambda_{2} \leqq 1$. In the present paper we shall consider results concerning products of functions representable in the form (1) which are consequences of the identity

$$
\begin{aligned}
& \left(1-D a^{-1}\right) f\left(\lambda_{1} x\right) g\left(\lambda_{2} x\right)=\lambda_{2} f\left(\lambda_{1} x\right)\left[g\left(\lambda_{2} x\right)-a^{-1} g^{\prime}\left(\lambda_{2} x\right)\right] \\
& \quad+\lambda_{1} g\left(\lambda_{2} x\right)\left[f\left(\lambda_{1} x\right)-a^{-1} f^{\prime}\left(\lambda_{1} x\right)\right]+\left(1-\lambda_{1}-\lambda_{2}\right) f\left(\lambda_{1} x\right) g\left(\lambda_{2} x\right) .
\end{aligned}
$$

We recall the definitions $\alpha_{1}=\operatorname{Max}_{a_{k}<0}\left[a_{k},-\infty\right], \alpha_{2}=\operatorname{Min}_{a_{k}>0}$ $\left[a_{k}, \infty\right]$, see $[2 ; 1]$.

Theorem 1. Let $G(x) \in$ class I and let

$$
f(x)=\int_{-\infty}^{\infty} G(x-t) d \alpha(t), \quad g(x)=\int_{-\infty}^{\infty} G(x-t) d \beta(t)
$$

with $\alpha(t) \in \uparrow, \beta(t) \in \uparrow$. If $0<\lambda_{1}, 0<\lambda_{2}, \lambda_{1}+\lambda_{2} \leqq 1$, then

$$
f\left(\lambda_{1} x\right) g\left(\lambda_{2} x\right)=\int_{-\infty}^{\infty} G(x-t) d \gamma(t)
$$

with $\gamma(t) \in \uparrow$.

\footnotetext{
${ }_{1}^{1}$ The first number in brackets refers to the references cited at the end of the paper. The number following the semicolon gives the section number of the reference cited.
}

Received by the editors November 21, 1949 and, in revised form, December 19 , 1949. 
PROOF. By $[3 ; 36]$ necessary and sufficient conditions for $h(x)$ to have the representation

$$
h(x)=\int_{-\infty}^{\infty} G(x-t) d \delta(t)
$$

with $\delta(t) \in \uparrow$ are:
a.
$h(x) \in C^{\infty}$
$(-\infty<x<\infty)$,
b.
$h(x)=o\left(e^{\alpha_{1} x}\right)$
$(x \rightarrow-\infty)$
$=o\left(e^{\alpha_{2} x}\right)$
$(x \rightarrow+\infty)$,
c.

$$
\prod_{j=1}^{n}\left(1-D A_{j}^{-1}\right) h(x) \geqq 0 \quad(-\infty<x<\infty)
$$

for every selection $\left\{A_{1}, A_{2}, \cdots, A_{n}\right\}$ from $\left\{a_{1}, a_{2}, \cdots\right\}$. Our assumptions imply that $f(x)$ and $g(x)$ fulfill these conditions; it follows that $f\left(\lambda_{1} x\right) g\left(\lambda_{2} x\right)$ also satisfies these conditions ( $\mathrm{a}$ and $\mathrm{b}$ trivially, and $\mathrm{c}$ because of (3)). Our desired result follows.

The following theorem may be proved similarly. See $[3 ; 36,37]$.

Theorem 2. Let $G(x) \in$ class II or III and let

$$
\begin{array}{ll}
f(x)=\int_{-\infty}^{\infty} G(x-t) d \alpha(t) & \left(x>r_{1}\right), \\
g(x)=\int_{-\infty}^{\infty} G(x-t) d \beta(t) & \left(x>r_{2}\right)
\end{array}
$$

with $\alpha(t) \in \uparrow, \beta(t) \in \uparrow$. If $0<\lambda_{1}, 0<\lambda_{2}, \lambda_{1}+\lambda_{2} \leqq 1$, then

$$
f\left(\lambda_{1} x\right) g\left(\lambda_{2} x\right)=\int_{-\infty}^{\infty} G(x-t) d \gamma(t) \quad\left(x>\operatorname{Max}\left[r_{1} / \lambda_{1}, r_{2} / \lambda_{2}\right]\right)
$$

where $\gamma(t) \in \uparrow$.

Using a further representation theorem $[3 ; 32]$ one may establish

THEOREM 3. Let $p, q$, and $r$ be numbers greater than one such that $r^{-1}=p^{-1}+q^{-1}$, and let

$$
\begin{aligned}
& f(x)=\int_{-\infty}^{\infty} G(x-t) e^{-c_{1} t} \phi(t) d t, \\
& g(x)=\int_{-\infty}^{\infty} G(x-t) e^{-c_{2} t} \psi(t) d t,
\end{aligned}
$$

where $\alpha_{1}<c_{1}<\alpha_{2}, \alpha_{1}<c_{2}<\alpha_{2}$, and where $\phi(t) \in L_{p}(-\infty, \infty), \psi(t)$ 
$\in L_{q}(-\infty, \infty)$. If $0<\lambda_{1}, 0<\lambda_{2}, \lambda_{1}+\lambda_{2} \leqq 1$, then

$$
f\left(\lambda_{1} x\right) g\left(\lambda_{2} x\right)=\int_{-\infty}^{\infty} G(x-t) e^{-c t} \chi(t) d t
$$

where $c=\lambda_{1} c_{1}+\lambda_{2} c_{2}$, and where $\chi(t) \in L_{r}(-\infty, \infty)$. More precisely we have

$$
\|\chi(t)\|_{r} \leqq E\left(c_{1}\right)^{-1} E\left(c_{2}\right)^{-1} \lambda_{1}^{-1 / p} \lambda_{2}^{-1 / q}\|\phi(t)\|_{p}\|\psi(t)\|_{q} .
$$

As an application of Theorem 1 we have the following result concerning the Stieltjes transform. If

$$
A(x)=\int_{0+}^{\infty}(x+t)^{-1} d \alpha(t), \quad B(x)=\int_{0+}^{\infty}(x+t)^{-1} d \beta(t)
$$

where $\alpha(t) \in \uparrow, \beta(t) \in \uparrow$, and if $0<\lambda_{1}, 0<\lambda_{2}, \lambda_{1}+\lambda_{2} \leqq 1$, then

$$
A\left(x^{\lambda_{1}}\right) B\left(x^{\lambda_{2}}\right)=\int_{0+}^{\infty}(x+t)^{-1} d \gamma(t)
$$

with $\gamma(t) \in \uparrow$. Choosing $A(x)=B(x)=x^{-1+\epsilon}, 0<\epsilon<1$, we see that the condition $\lambda_{1}+\lambda_{2} \leqq 1$ is essential.

\section{REFERENCES}

1. I. I. Hirschman, Jr., and D. V. Widder, An inversion and representation theory for convolution transforms with totally positive kernels, Proc. Nat. Acad. Sci. U.S.A. vol. 34 (1948) pp. 152-156.

2. - The inversion of a general class of convolution transforms, Trans. Amer. Math. Soc. vol. 66 (1949) pp. 135-201.

3. - A representation theory for a general class of convolution transforms, Trans. Amer. Math. Soc. vol. 67 (1949) pp. 69-97.

WASHINGTON UNIVERSITY AND HARVARD UNIVERSITY 